\title{
Research of Electrical Engineering Specialty Experiment Teaching based on MATLAB
}

\author{
Xiujuan Qie ${ }^{\mathrm{a}}$, Huanna LI $^{\mathrm{b}}$, Jiao Wang ${ }^{\mathrm{c}}$ \\ Department of Electrical and Mechanical Engineering, HeBei College Of Science And Technology, \\ China \\ a942711710@qq.com, ${ }^{\mathrm{b}} 124884677 @ q q . c o m,{ }^{\mathrm{c}} 1151620819 @ q q . c o m$
}

Keywords: Simulation; Simulation results; MATLAB

\begin{abstract}
The use of MATLAB's powerful computing power and graphics capabilities and simulink module library may carry out simulation results to most of the electrical engineering specialty curriculum. For example, in circuit analysis, electronics, control systems such curriculum experiment. Through the preparation processor the establishment of simulink model, obtaining simulink experimental data and its analysis. Thus makes better combination of theoretical knowledge and practice, further inspires students to think-solving skills, problem-solving ability and stimulate greater interest in students learning.
\end{abstract}

\section{Introduction}

With the progress of the development of science and technology, computer technology, colleges and universities are required to develop "practical talents, creative talent" as the teaching goal. But the realization of this goal is inseparable from the practical aspects of teaching, because the students can improve the abilities of practicing, observing and soling problems through hands-on experiments. Electrical engineering has been developed for several years, most of the original courses discrete components assembled experiments have been replaced by digital experimental platform and experimental box [1]. The students understand the contents of principle experiment, even if not enough, just read the guide books and under the teachers' guidance of a mechanical connection and reading, and then apply the formula to analyze the experimental data to complete the test content. This will cause the students' theoretical knowledge and practical divorced, so that students practical ability weakened, which led to their awareness of knowledge innovation is weak. For such problems, we can use computer software development, simulation experiments, and the software can select MATLAB language which is based on a complex matrix as the basic programming unit of a high-level programming language, not only provides a variety of matrix arithmetic, strong graphics and a large number of simulation module libraries, but other languages can implement interfaces. This software is now widely used in engineering calculations, image processing, simulation, and other fields. The application of MATLAB simulation experiments requires students to have a deeper theoretical knowledge, the only way to compile the correct procedures or to establish the correct mathematical model, complete simulation experiment on this basis, re-yourself hardware experiment teaching not only saves time but also can better achieve the combination of theory and practice, teaching can play a multiplier effect [2].

\section{MATLAB in Circuit Analysis}

Fig. 1 is a full-response analysis of experimental RLC circuit. The circuit is a second order circuit, the analysis need to be listed in the usual way to solve differential equations. Moreover, the calculation process is complex, and the use of artificial depiction response curves plotted point method is difficult to ensure its accuracy degree. If it is simulated using MATLAB software, it will have simple computation, high accuracy and so on. 


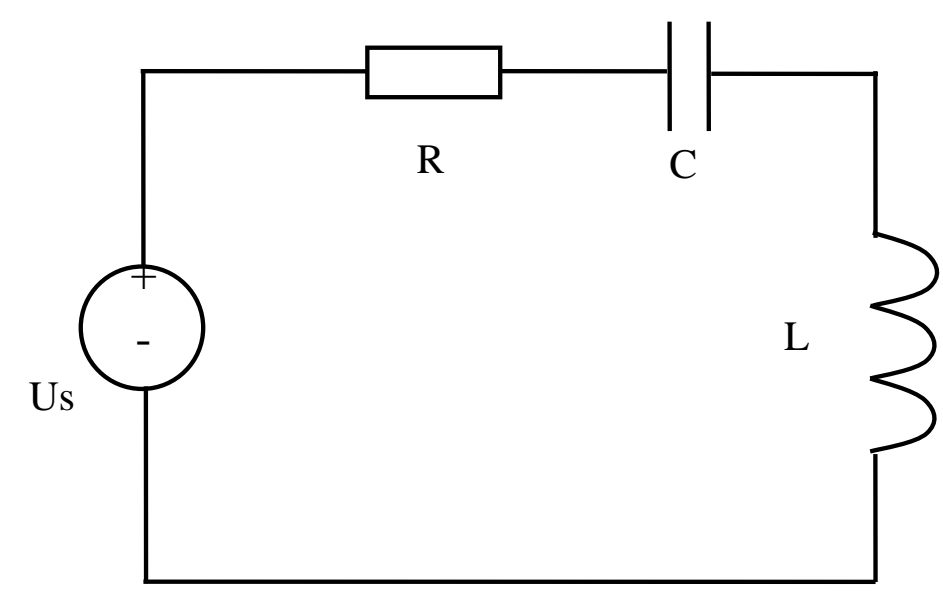

Fig.1 RLC circuit diagram of a second-order dynamic

The differential equations of Fig. 1 are shown as follows:

$$
\begin{aligned}
& L C \frac{\mathrm{d}^{2} U_{c}}{\mathrm{~d}^{2} t}+R C \frac{\mathrm{d} U_{c}}{\mathrm{~d} t}+U_{c}=U_{s} \\
& U_{c}(t)=k_{1} e^{s_{1}(t)}+k_{2} e^{s_{2}(t)}+U_{s}
\end{aligned}
$$

Where $s_{1,2}=-\frac{R}{2 L} \pm j \sqrt{\frac{1}{L C}-\left(\frac{R}{2 L}\right)^{2}}, k_{1,2}=\frac{1}{s_{1}-s_{2}}\left\{s_{1,2}\left[U_{c}-U_{s}\right]-\frac{i_{L}\left(0_{+}\right)}{C}\right\}$.

If the following parameters are as known: $R=10 \Omega, L=2 \mathrm{H}, C=100 \mu \mathrm{F}, U_{c}=\left(0^{+}\right)=0.5 \mathrm{~V}$, $i_{L}\left(0^{+}\right)=1 \mathrm{~mA}$ and $U_{s}=1 \mathrm{~V}$, MATLAB is used to simulate, and the full-response curve is shown in Fig.2.

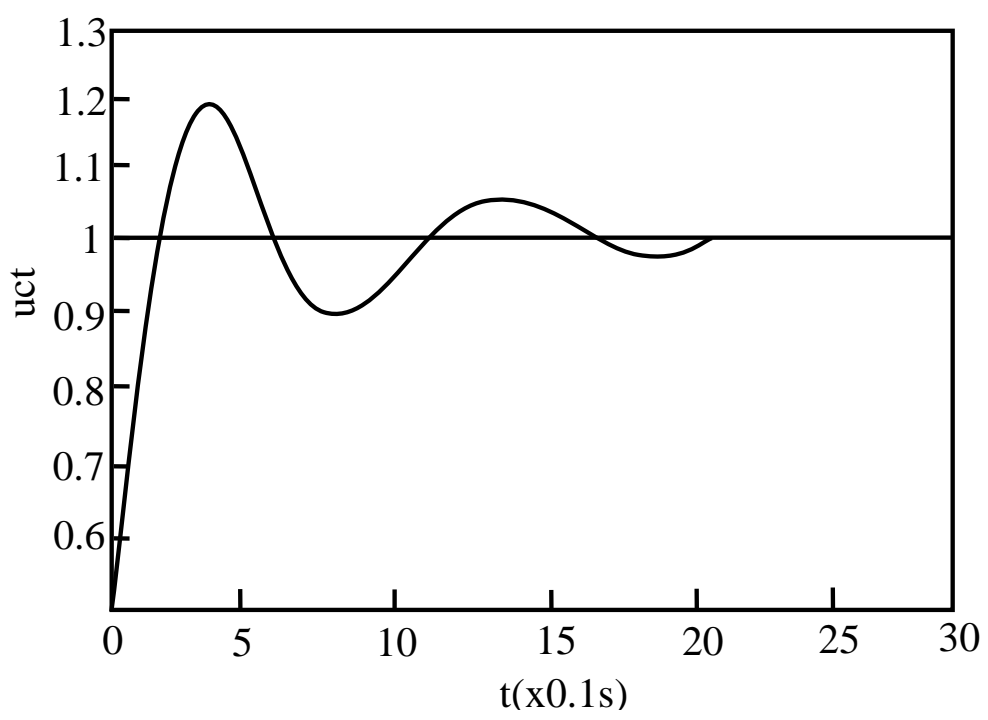

Fig.2 The full-response curve

The MATLAB code is listed as follows:

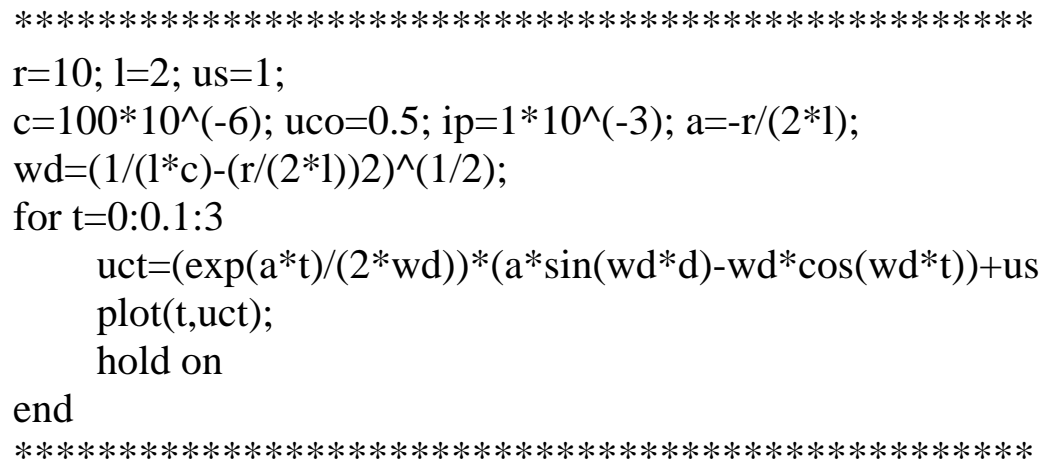

The above example illustrates that the circuit analysis experiment teaching is introduced if 
MATLAB can improve the accuracy and efficiency, making the theory more realistic, but also to improve the experimental teaching mode circuit analysis intelligent platform to complete the experiment.

\section{MATLAB in electronic technology experiment teaching}

Electronic technology including analog electronics and digital electronics, which contains a large number of experiments. If the MATLAB Simulink block library is used to simulate experiments, it will be better for students to understand the basic principles and application value. This paper takes the the diode limiter simulation experiment as an example, the concrete description is shown as follows:Diode limiter analog experiment is relatively simple experiment in electronic technology. Its data can be readily available through hardware experiment, but if you want to learn more about the process of clipping diodes and get the output voltage waveform, simple hardware experiment is difficult to complete. Thus, the module of "Power Electronics" in Simulink block library can be used to complete the simulation. The bidirectional limiter diode is shown in Fig.3, and its corresponding simulation model is shown in Fig.4.

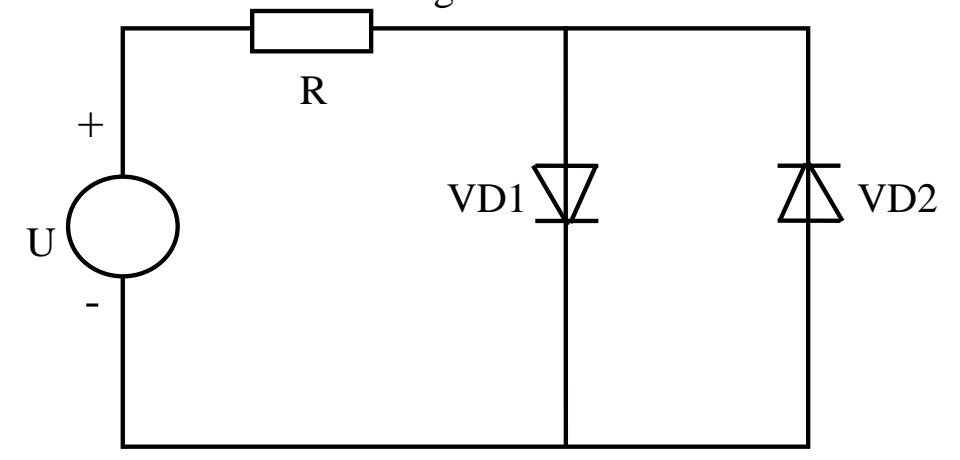

Fig.3 Bidirectional diode limiter circuit

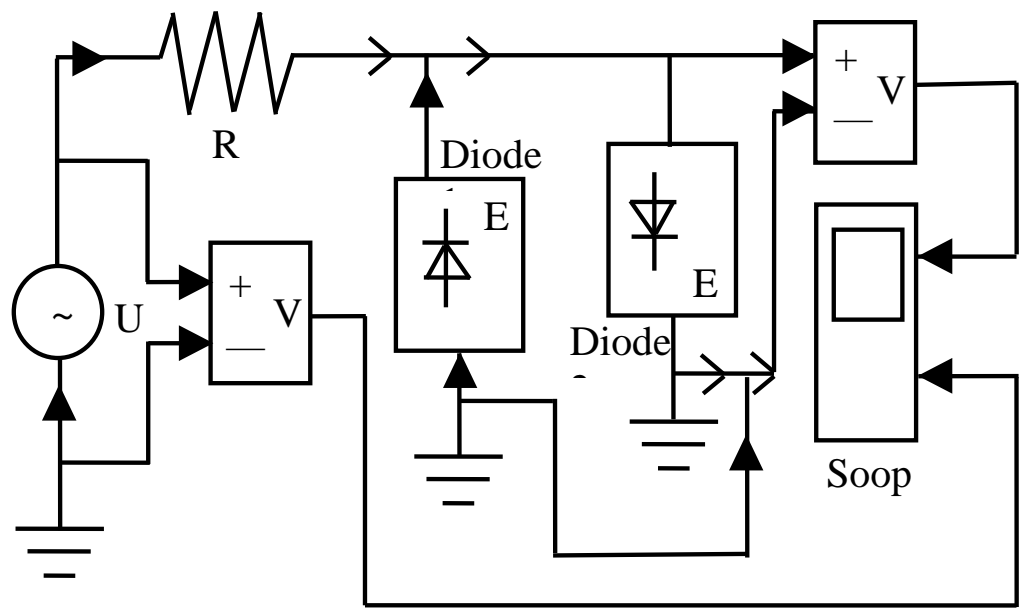

Fig.4 Bidirectional diode limiter circuit simulation model

Set $u=3 \sin t$ and $R=2 \Omega$. Diode parameters are shown in Table 1 .

Table 1 Diode module parameter settings

\begin{tabular}{ccc}
\hline Paremeters & Diode1 & Diode2 \\
\hline Resistance Ron & 0.01 & 0.01 \\
Inductance Lon & 0 & 0 \\
Snubber resistance & Inf & Inf \\
Forward voltage & 0.7 & 0.7 \\
Snubber capacitance & 0 & 0 \\
Initial current & 0 & 0 \\
\hline
\end{tabular}

The voltage waveform and the power waveform on a-side and b-side can be obtain which is shown in Fig.5. As can be seen from Fig.5, the voltage source output is described as a sine wave 
whose amplitude is three, and the voltage of a-side and b-side are limited to a $0.7 \mathrm{~V}$.

$\mathrm{u}$

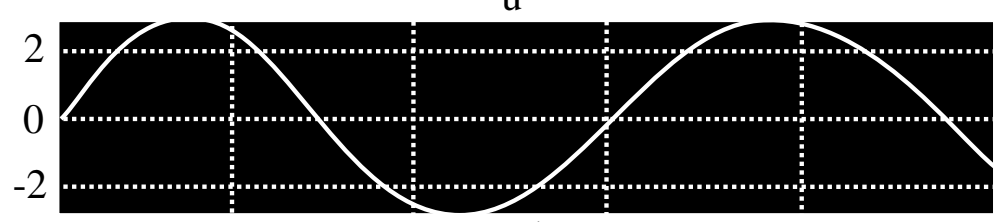

uab

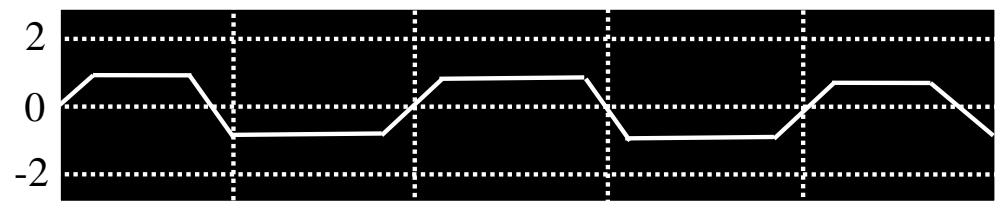

Fig.5 Voltage output waveform

\section{Design and simulation in control system based on MATLAB}

Development of control theory is broadly divided into three stages: the classical control theory, modern control theory and intelligent control theory [3]. The analysis and design of these theories requires a lot of mathematical calculations and a variety of analytical methods. If provided by means of MATLAB Control System Toolbox can greatly improve the efficiency of control systems analysis and design.

Control System Toolbox is a MATLAB software designed specifically for the control system engineering functions and a collection of tools. It mainly uses $\mathrm{M}$ file format, and is used to analyze the feedback control system design and simulation, which covers the classical control theory and modern control theory of large part. In addition, the proposed toolbox applies for both continuous system and discrete systems, and can achieve mutual conversion between different models. To determine the controllability and observability of the system is better to prepare a simple M-file and use two functions $\operatorname{ctrb}($ ) and $\operatorname{obsv}($ ) to complete.

For example, the known dynamic equation system is as follows:

$$
\begin{gathered}
{\left[\begin{array}{l}
x_{1} \\
x_{2}
\end{array}\right]=\left[\begin{array}{cc}
-4 & 1 \\
0 & -2
\end{array}\right]+\left[\begin{array}{l}
2 \\
1
\end{array}\right] u} \\
y=\left[\begin{array}{ll}
1 & 3
\end{array}\right]\left[\begin{array}{l}
x_{1} \\
x_{2}
\end{array}\right]
\end{gathered}
$$

Determine the system controllability and observability, the MATLAB codes are as follows:

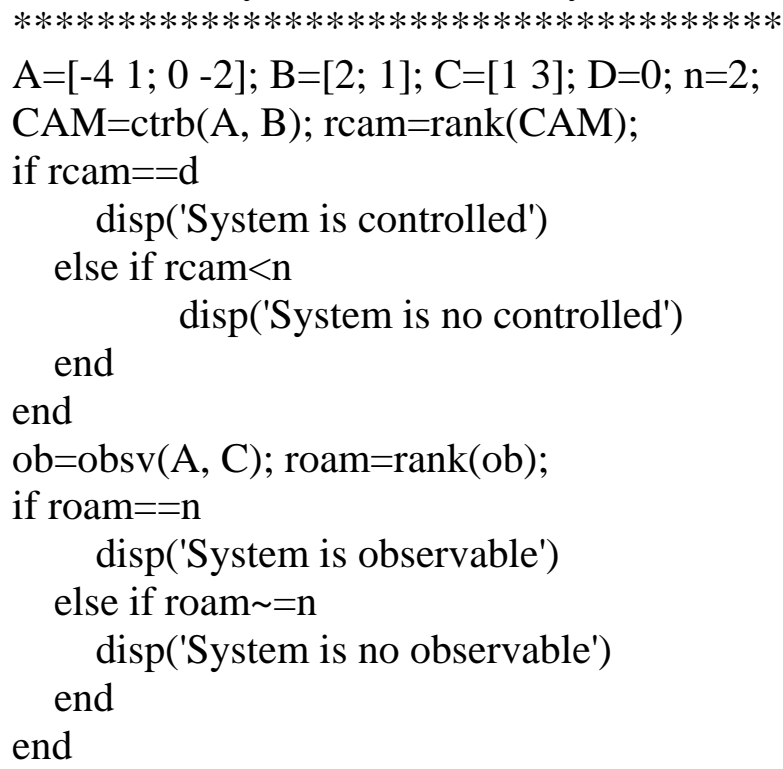

$* * * * * * * * * * * * * * * * * * * * * * * * * * * * * * * * * * * * * *$ 
The result of the program is "System is controlled; System is observable". From the results, the system is controllable and observable. Using MATLAB can also complete time domain analysis, root locus analysis, frequency domain analysis, design and other PID controller. With control continuous development and deepening of the study of theory, MATLAB will make the design and analysis of control systems more simple and easy, and can be designed more sophisticated control systems.

\section{Conclusions}

Since MATLAB toolbox has the advantages of ease of usage, reliability, versatility and professionalism, it has been used by more and more people, not only can be used in circuit analysis, electronic technology, control systems, it can also be used in power electronics more curriculum areas as well as digital signal processing. With the development of computer technology and digital technology, which in engineering universities has been widely used, not only is electrical engineering, other areas such as electronic communications, electronics, computers and other professional software can also be used to complete the course of the simulation, so to achieve full use of computer teaching resources, but also the course consists of theoretical analysis to the analysis of the actual technical issues forward, try to make students learn the theoretical expertise to keep pace with scientific and technological development.

\section{References}

[1] Cao Jinfang, Jin Ruojun, Jiang Yinong and Gao Jiping. Teaching Experiences of Servo System Experiments. Advances in Control Education. Vol. 3 (1995), p. 13-15.

[2] Alexandru Baloi, Adrian Pana, Florin Molnar-Matei. Advantages of Using MatLab Simulink in Laboratory Lessons on Operating Conditions of Overhead Power Lines. Procedia - Social and Behavioral Sciences. Vol. 191(2015), p. 179-184.

[3] Christian Eugène. How to teach at the university level through an active learning approach Consequences for teaching basic electrical measurements. Measurement. Vol. 39 (2006), p. 936-946. 